\title{
Novel Battery-Less Sustainable Energy Harvester Scheme for On-board Electronic Units
}

\author{
Dr. G. Ranganathan, \\ Head of the department, \\ Department of Electronics \& Communication Engineering, \\ Gnanamani College of Technology, \\ Namakkal, India.
}

\author{
Dr. Bindhu V, \\ Professor and Head, \\ Department of Electronics and Communication Engineering, \\ PPG Institute of Technology, \\ Villankurichi Saravanampatti P.O, \\ Coimbatore, India.
}

\begin{abstract}
With the rapid development in smart vehicles, the on-board unit is established for communication between the vehicle and toll collection booth for toll collection. However, providing a continuous power supply to such units has been a challenge. While features like compactness, low cost and simplicity are to be maintained, the accuracy and performance of the device are to be maintained intact. This paper proposes a battery-less energy harvester scheme as a solution to this power issue. Maximum energy transmission Several researches are conducted on obtaining maximum energy transmission through performance optimization in this domain. This paper provides maximum performance while minimizing the energy harvester transponder dimensions. The energy transmitted to the transponder is maximized considering the optimal source impedance. This provides power to the battery-less structure. The radio frequency to DC rectifier is provided with an optimal source impedance with the help of a small patch antenna. Utilization of a lossy matching network can be avoided through this technique. Inter-stage matching network is used for comparison of the function. The energy efficiency using the proposed scheme is increased by a factor of $10 \%$ when compared to the existing schemes.
\end{abstract}

Keywords: Battery less, rectifier, power management, efficiency, energy harvesting;

\section{Introduction}

With the rapid development in Internet of Things (IoT), several solutions for intelligent transportation systems (ITS) are provided using this technology [1]. The mobile application and communication technology limitations are overcome with the help of IoT. Applications like traffic efficiency, road safety and utilities can make use of this technology at a larger scale. Electronic toll collection is a relevant utility based application where this technology can be implemented. Vehicle identification, assets control, parking payment, and e-payment features can be linked with this system. The on-board and roadside units are designed based on the application [2]. Fundamental requirements like high reliability, low complexity and lesser cost are provided by this system. However, with the development of $5 \mathrm{G}$ technology, meeting the severe requirements in these units is challenging. In order to reduce the complexity and cost while improving the performance of the model, technological evolution is essential. Battery-less solution offers increased diffusion, reduced logistic chains, decreased cost and simplified architecture for this system. The system can operate at very low power supply while utilizing all the required electronic components using batter-less model. The existing battery-less approaches can be implemented only in applications with reduced power requirement and lower complexity that offers low performance [3].

Ambient sources such as RF waves, thermal, kinetic or solar can be used for conversion of energy into electrical energy that is required for powering the batteryless IoT system without human interference [4]. In order to utilize the batteryless IoT system in an extensive manner, energy harvesting approaches can be used. The motion, ambient vibration and mechanical energy can be converted into electrical energy with the help of kinetic energy harvesting technique in batteryless IoT. The harvested energy is utilized for powering the load by the Power Management Unit (PMU) [5]. The sensor node is powered using an energy storage unit that contains the harvested energy. The transceiver, microcontroller, Analog-to-Digital Converter (ADC), sensor module and other hardware components of the sensor node consume the harvester energy while sampling the physical attributes and transmitting information to the server or destination. Energy Neutral Operation (ENO) is established when the harvested energy 
Journal of Electrical Engineering and Automation (EEA) (2020)

Vol. 02/ No. 03

Pages: 135-140

https://www.irojournals.com/iroeea

DOI: https://doi.org/10.36548/jeea.2020.3.005

is sufficient for powering the sensors. If not, an external depletable energy source is used for powering the system [6]. The transport modes and human activities in IoT applications that are based on human-centric sensors make use of piezoelectric transducers as powering solutions. Transducer must operate at its maximum power point (MPP) for the purpose of harvesting maximum energy.

\section{Literature Review}

With emerging technologies in industrial internet, supply chain management, precision agriculture, automotive, healthcare, smart manufacturing, smart cities and home automation, low power consumption and autonomous systems have been receiving great attention [7]. For continuous operations of these applications, various energy harvesting schemes must be incorporated to cope up with the limited power sources in the network infrastructure. Research is conducted on several self-sustaining power sources for application in wireless embedded technologies. Applications involving environmental sensor networks with an energy based upgradable platform using solar harvesting is presented by certain researchers [8]. Energy storage is performed in rechargeable NiMH batteries along with solar panels that act as a power source in these devices. Cheap energy storage units and simple hardware structure is featured in this design. The power supply is analyzed and its charging and discharging characteristics are studied.

A plug-and-play solar energy harvesting module is presented where performance evaluation is done following the design and implementation [9]. The authors use Berkeley/Crossbow sensor nodes for powering the module. A rechargeable battery and energy harvesting circuit is available in the module. The power routing, energy storage and energy harvesting decisions are managed autonomously. Instantaneous data is provided regarding the battery state and solar energy by the harvesting-aware operation [10]. Agricultural applications and environmental monitoring can also be performed using outdoor sensor networks. Temperature sensor, flash memory, real-time clock, regulator, on-board battery charging circuit, solar panel, primary power source using rechargeable NiMH batteries and Nordic nRF905 RF transceiver are the components incorporated in this platform. The charging current, charging voltage, battery current and battery voltage can be monitored using this module. The energy generated and consumed are both computed using the platform's power input stage. The distributed energy-aware applications can be implemented using this technique [11].

The power sources are routed to the subsystem using a power distribution switch and the power from ambient power source and its total utility is maximized using the power source to consumption matching algorithm [12]. In this system, the ambient energy intensity is detected by an array of sensors. The power source utilization is maximized by the controller algorithm. Further, connections are established between the subsystems and power sources with the help of switch array. Power defragmentation and maximum power point tracking (MPPT) are combined to obtain higher ambient power utility. Light sensors controlled using microcontrollers are also used. Supercapacitors can be used for durable energy storage integrated with small-scale systems and sub-watt to provide feasible energy harvesting modules in wireless sensor nodes [13]. The supercapacitors are charged with pulse frequency modulated feed-forward regulator at near-optimal operating points for solar cells. The supercapacitors are charged with the solar cell based energy. The available energy is maximized by efficient charging of supercapacitors with the help of solar cells and MPPT circuit. The MPPT algorithm is run by the microcontroller in the circuit. The lifetime of sensor devices can be maximized by implementing energy transfer for perpetual operation. A rechargeable lithium battery, two-stage storage unit consisting of supercapacitors, a charging circuit and solar panel are used in this system.

\section{Proposed Work}

The design principle of the proposed harvester block is represented in Figure 1. The impedance required is transmitted to the RF-to-DC conversion block from the optimal impedance matching network. Further, a tank capacitor, DC/DC boost, RF-to-DC rectifier and optimal impedance matched antennas aids energy storage in the proposed model. 


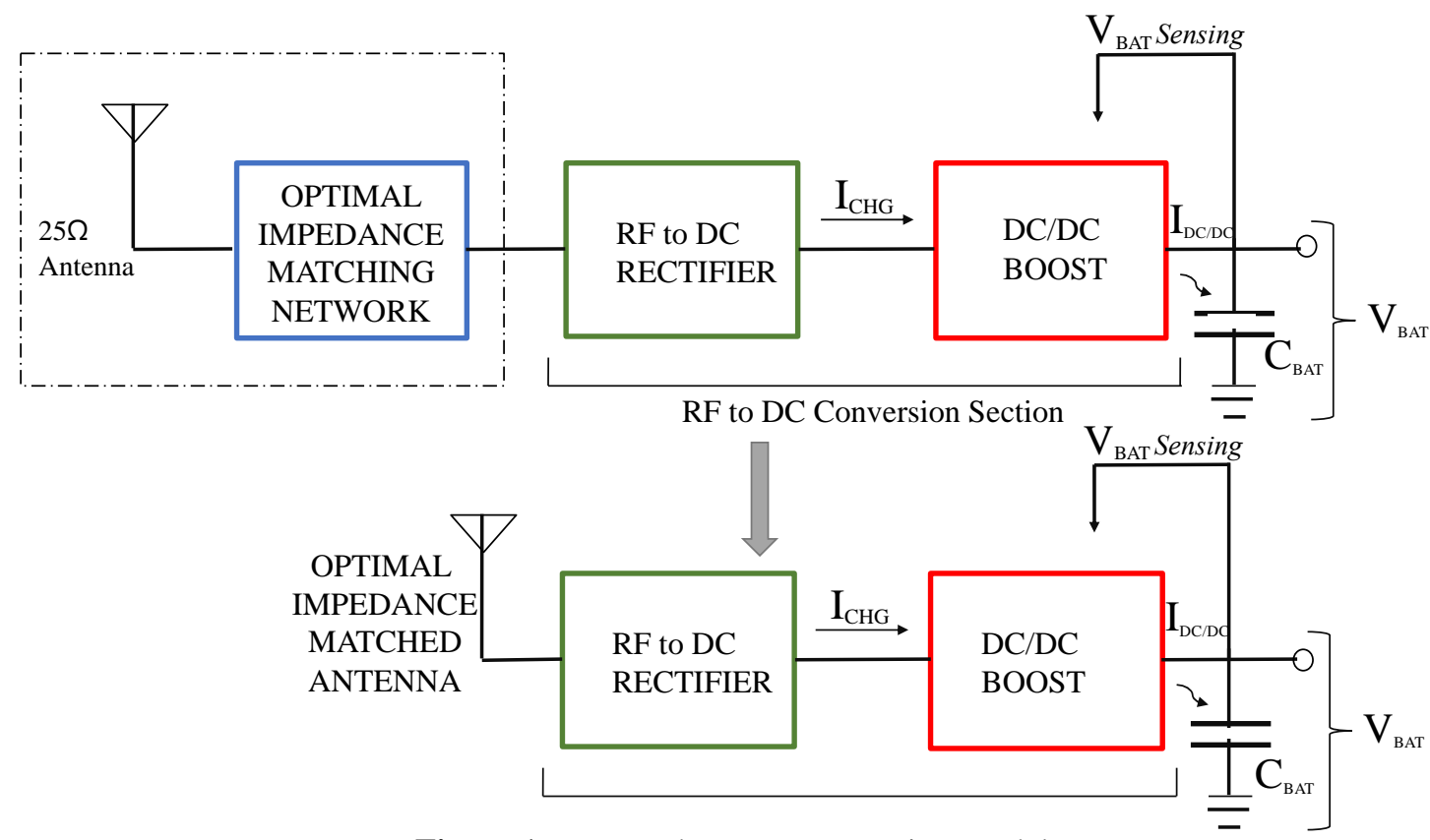

Figure 1: Proposed Energy Harvesting Model

The DC/DC converter blocks and RF-to-DC rectifier are cascaded in the sub-system considered for analysis. RF to DC conversion is performed using a frequency power converter made of RF-to-DC rectifier created by cascading the blocks as represented in Figure 2. Nonlinear techniques are applied for analysis of the harvester block as per the requirement of the power converter and its intrinsically nonlinear behavior. RF-DC output current $\mathrm{I}_{\mathrm{CGH}}$ is increased by maximizing the performance of the harvester thereby feeding the DC to DC boost. This inturn reduces the DC to DC charge time. The buck conversion harvester module is used for implementation and evaluation of the DC to DC boost along with a variable input termination scheme.

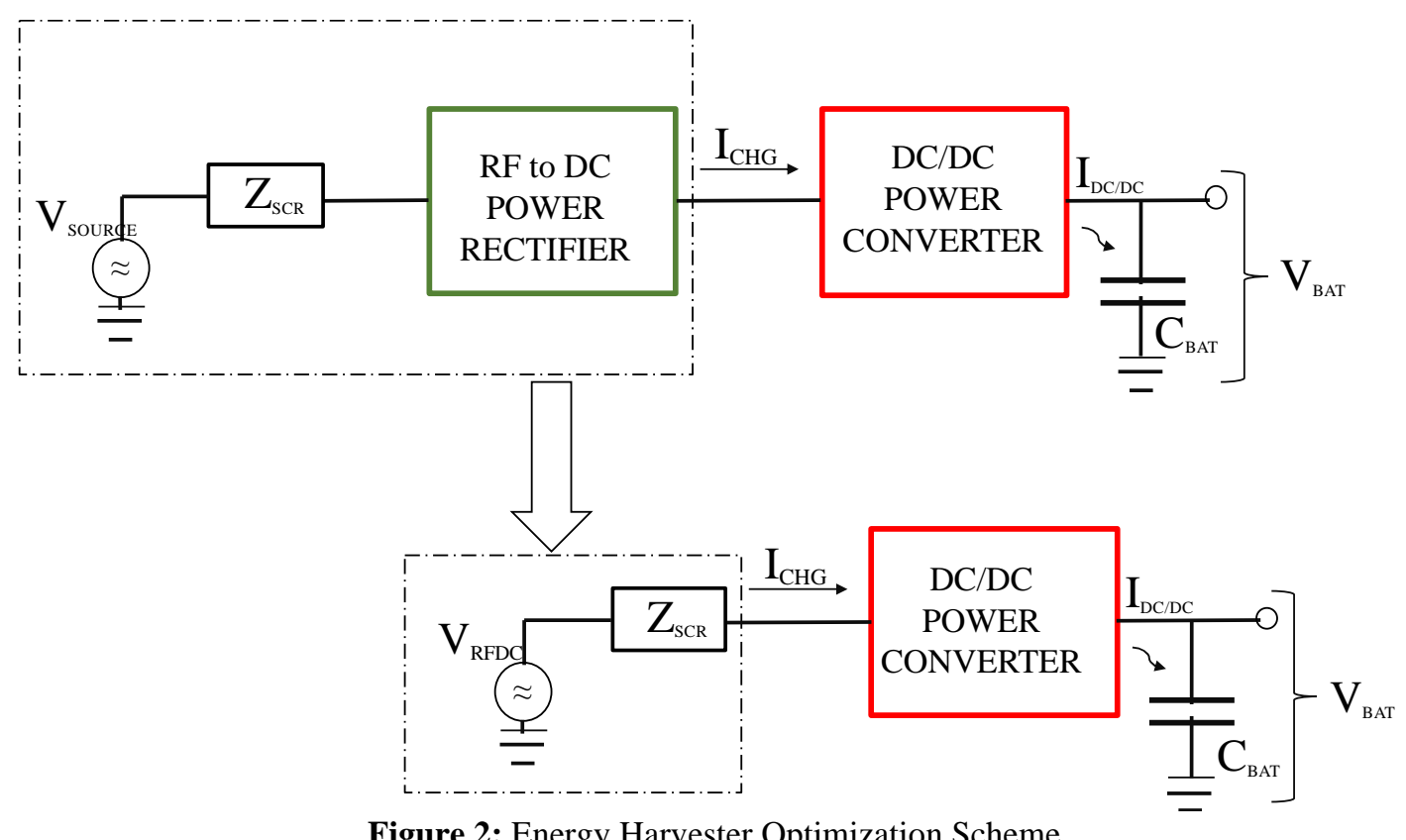

Figure 2: Energy Harvester Optimization Scheme

Simulation is performed to represent the variable input termination in the DC to DC block. Maximum possible current is drained by the boost converter available in the DC to DC block. A non-zero output resistance is exhibited by the input source. This results in reduced input voltage with increased current absorption. The state 
Journal of Electrical Engineering and Automation (EEA) (2020)

Vol. 02/ No. 03

Pages: 135-140

https://www.irojournals.com/iroeea

DOI: https://doi.org/10.36548/jeea.2020.3.005

parameters of DC to DC power conversion also varies consequently. $Z_{\mathrm{SCR}}$ represents the source pull of the impedance algorithm. $\mathrm{V}_{\mathrm{BAT}}$ and $\mathrm{C}_{\mathrm{BAT}}$ represents the battery voltage and capacitance respectively.

\section{Results and Discussion}

Simulation is performed to analyze the various impedance performance characteristics, antenna pattern and other parameters of the proposed model. Figure 3 represents the reflection coefficient of the $25 \Omega$ antenna represented in the proposed model. $5.8 \mathrm{GHz}$ frequency range offers the optimal operational condition. The operation of the proposed harvester is around a narrowband center frequency. Between 5.7 to $5.9 \mathrm{GHz}$ range, the $10 \mathrm{~dB}$ return loss can be noticed. This approves the efficiency of the proposed model without the need for a matching network.

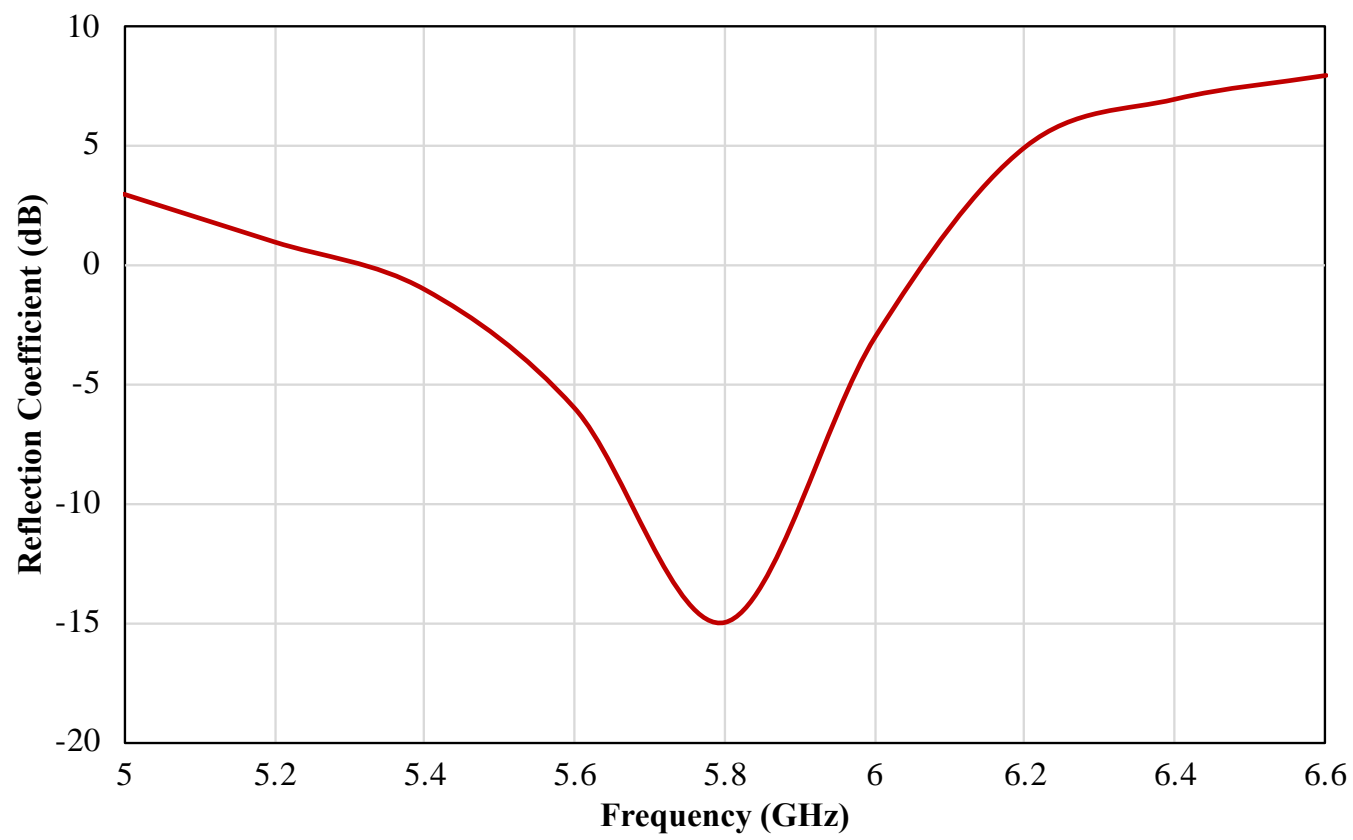

Figure 3: Simulation of reflection coefficient

The radiation pattern of the simulated antenna can also be studied. The impinging power and the corresponding charge current measured is represented in figure 4 . The transmission power is varied in subsequent iterations to analyze the performance of the proposed model. Towards the boresight direction, a $6.6 \mathrm{~dB}$ gain is achieved. The antenna radiative performance is not affected by the optimal impedance efficient performance outputs are seen for the proposed design conditions. This high efficiency is maintained as the external matching network is eliminated from the model. The harvester's impedance is also optimized in the proposed model. Good heuristic conditions are also tested while performing the final optimization. All available degrees of freedom are exploited to pursue the best match and gain parameters. The antenna radiative performance is maintained while the performance of the design conditions and optimal impedance and improved. 
Journal of Electrical Engineering and Automation (EEA) (2020)

Vol. 02/ No. 03

Pages: 135-140

https://www.irojournals.com/iroeea

DOI: https://doi.org/10.36548/jeea.2020.3.005

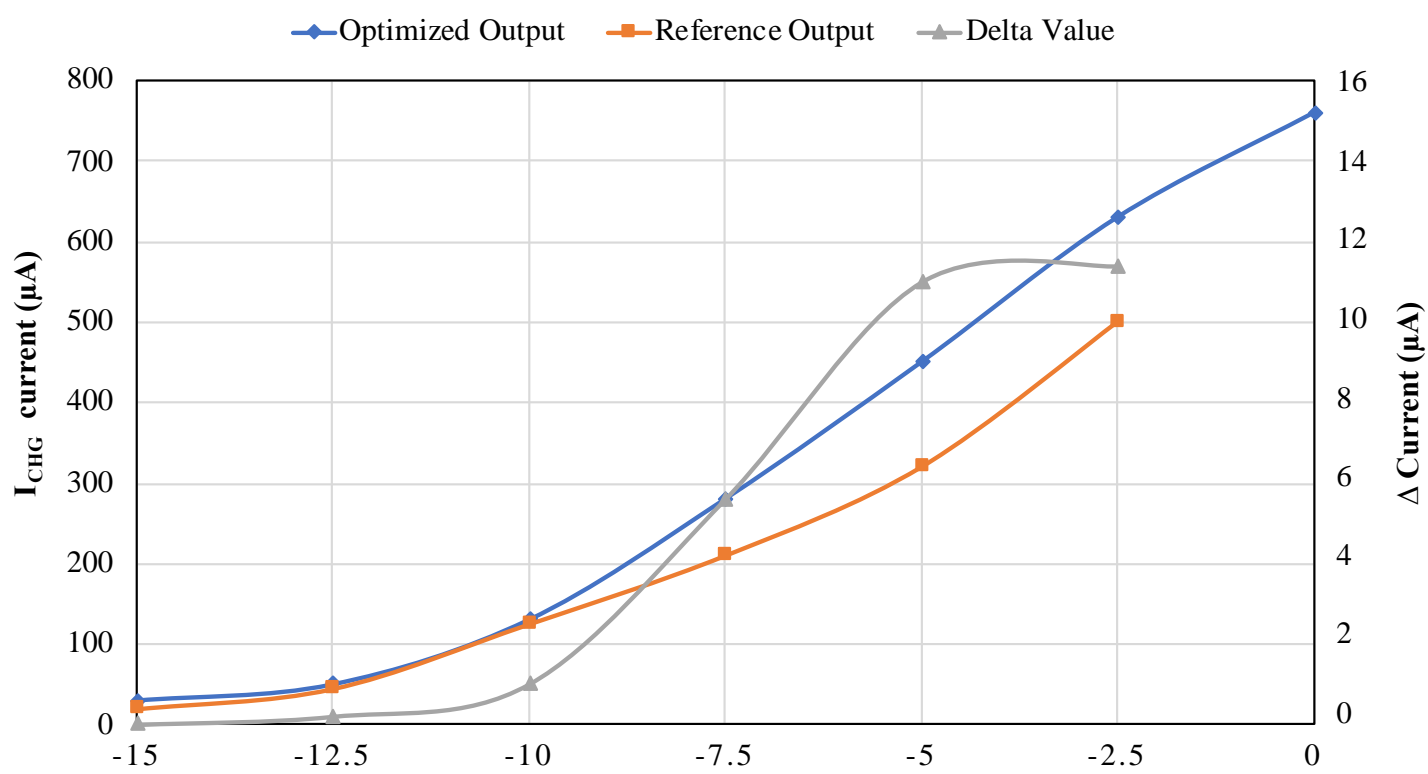

Figure 4: Impinging power versus charge current

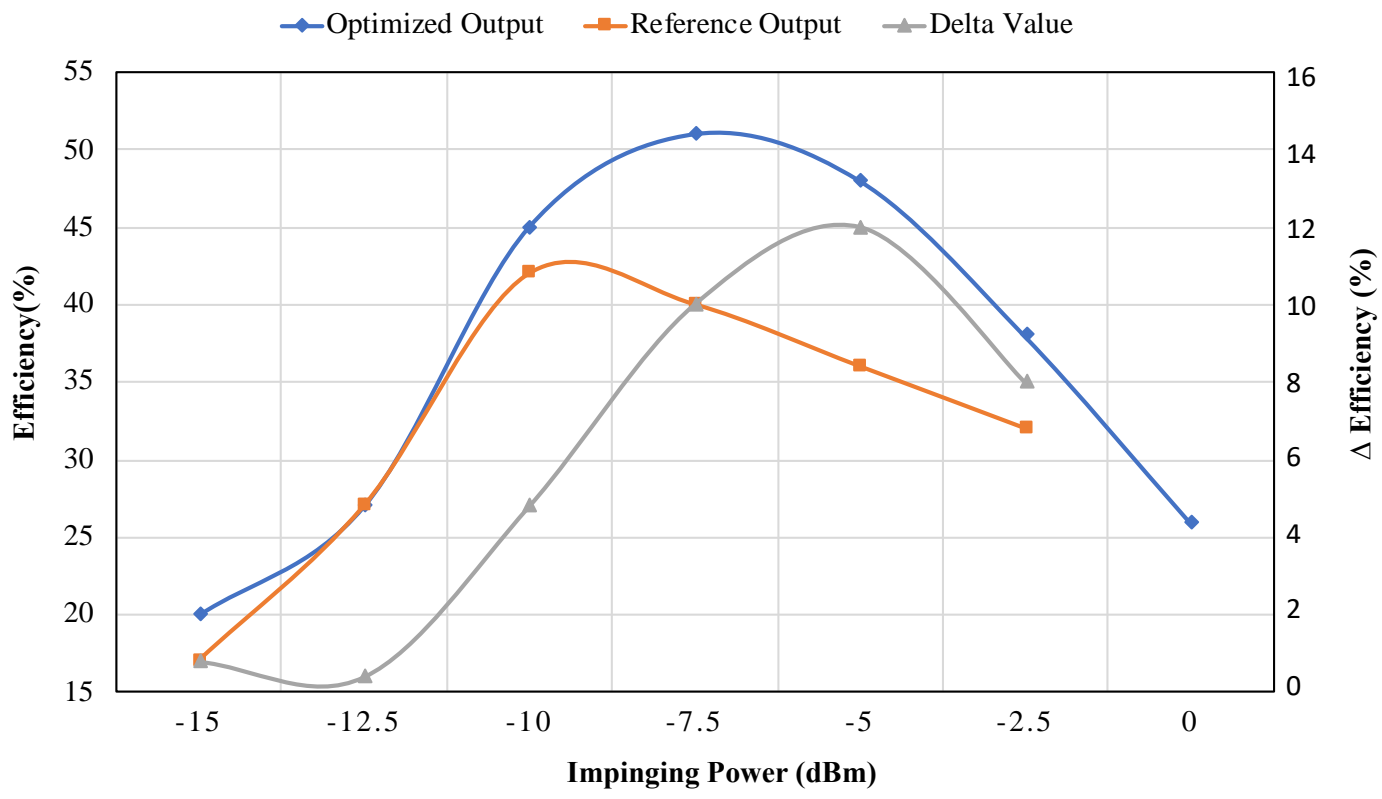

Figure 5: Impinging power versus conversion efficienct

Considering the impinging power, the reference prototype and harvester prototype conversion efficiency is compared. From figure 5 it is evident that lower impinging power levels exhibit similar behavior. The conversion efficiency is improved by $\sim 10.0 \%$ with the reference level of $-5 \mathrm{dBm}$ for the optimized impinging power in the proposed model. At the input of the rectifier, the impinging power and current is plotted across each other. The reference solution and the proposed solution offer similar outputs up-to the range of -10dBm. However, at higher values, the compact model offers improved efficiency and performance.

\section{Conclusion}

In this paper, an optimal module is designed providing solution to on-board vehicle communication module for energy harvesting. The complexity and cost of on board unit is analyzed and reduced. A $25 \Omega$ antenna is used for integration of the harvesting prototype. This offers gain similar to that when a matching network is employed. The proposed system offers enhanced performance output when compared to the existing model. The performance efficiency and charging current improves by $20 \%$ on simulation of the proposed module. The 
Journal of Electrical Engineering and Automation (EEA) (2020)

Vol. 02/ No. 03

Pages: 135-140

https://www.irojournals.com/iroeea

DOI: https://doi.org/10.36548/jeea.2020.3.005

impinging power level for optimization of the prototype is set at $-5 \mathrm{dBm}$. Better behavior is represented by the compact prototype at an impinging power of $-10 \mathrm{dBm}$ and higher. When compared to the charge current measured, the proposed model provides thrice the efficiency of the existing models.

\section{References}

[1] Kazmierski, T. J., \& Beeby, S. (2014). Energy harvesting systems (p. 2011). New York: Springer.

[2] Schmickl, S., Faseth, T., \& Pretl, H. (2020). An RF-energy harvester and IR-UWB transmitter for ultralow-power battery-less biosensors. IEEE Transactions on Circuits and Systems I: Regular Papers, 67(5), 1459-1468.

[3] Miyoshi, T., Adachi, M., Tanaka, Y., \& Suzuki, Y. (2018, July). Low-profile Rotational Electret Energy Harvester for Battery-less Wearable Device. In 2018 IEEE/ASME International Conference on Advanced Intelligent Mechatronics (AIM) (pp. 391-394). IEEE.

[4] Bradai, S., Bouattour, G., Naifar, S., \& Kanoun, O. (2020, June). Electromagnetic Energy Harvester for Battery-Free IoT Solutions. In 2020 IEEE 6th World Forum on Internet of Things (WF-IoT) (pp. 1-5). IEEE.

[5] Schmickl, S., Faseth, T., \& Pretl, H. (2020). An RF-energy harvester and IR-UWB transmitter for ultralow-power battery-less biosensors. IEEE Transactions on Circuits and Systems I: Regular Papers, 67(5), 1459-1468.

[6] Sabovic, A., Delgado, C., Subotic, D., Jooris, B., De Poorter, E., \& Famaey, J. (2020). Energy-Aware Sensing on Battery-Less LoRaWAN Devices with Energy Harvesting. Electronics, 9(6), 904.

[7] Alghisi, D., Ferrari, V., Ferrari, M., Crescini, D., Touati, F., \& Mnaouer, A. B. (2017). Single-and multisource battery-less power management circuits for piezoelectric energy harvesting systems. Sensors and Actuators A: Physical, 264, 234-246.

[8] Mekid, S., Bouhraoua, A., \& Baroudi, U. (2019). Battery-less wireless remote bolt tension monitoring system. Mechanical Systems and Signal Processing, 128, 572-587.

[9] Colaiuda, D., Leoni, A., \& Ulisse, I. (2020, January). RF Energy Harvester Rectifier Block Design Optimization: a Comparison with a Commercial Device. In 2020 International Conference on Mathematics and Computers in Science and Engineering (MACISE) (pp. 159-162). IEEE.

[10] Munir, B., \& Dyo, V. (2018). On the Impact of mobility on battery-less RF energy harvesting system performance. Sensors, 18(11), 3597.

[11] Qi, C., Frederick, Q., Davis, K., Lindsay, D., Cox, J., Parke, S., ... \& Durgin, G. D. (2018, December). A $5.8 \mathrm{GHz}$ energy harvesting tag for sensing applications in space. In 2018 6th IEEE International Conference on Wireless for Space and Extreme Environments (WiSEE) (pp. 218-223). IEEE.

[12] Sakya, S. (2020). Design of Hybrid Energy Management System for Wireless Sensor Networks in Remote Areas. Journal of Electrical Engineering and Automation (EEA), 2(01), 13-24.

[13] Sathesh, A. (2019). Optimized multi-objective routing for wireless communication with load balancing. Journal of trends in Computer Science and Smart technology (TCSST), 1(02), 106-120. 